\title{
Hacia la comprensión del espacio urbano global: ¿Sistemas de ciudades o Redes Urbanas? ${ }^{1}$
}

\author{
Julio A. del Pino Artacho *
}

\begin{abstract}
RESUMEN ABSTRACT
El presente trabajo aborda los estudios sobre las relaciones interurbanas de un modo general. Traza un camino por esta área señalando los dos desarrollos más sobresalientes que ha tenido. 1. Por un lado, el análisis de los sistemas de ciudades, con los conceptos de especialización funcional y jerarquia urbana. 2. Por otro, el análisis de las consecuencias urbanas de la globalización, con su doble vertiente de estudio, la ciudad global y la red global de nodos urbanos. 3. Sistemas y redes son dos formas de estructurar las relaciones interurbanas que difieren ampliamente. Ambas se dan, en alguna medida, en la realidad. Su exploración conceptual, con ánimo clarificador, sintético y comprensivo se ofrece para finalizar.

This paper tackles the interurban relationships in a comprehensive way. Two major approaches are shown: 1. On one hand, the analysis of the system of cities, with reference to functional specialization and urban hierarchy. 2. On the other hand, the analysis of the urban results of globalisation, with the study of the global city and the global network of urban nodes. 3. Systems and networks are two different ways of structuring the inter-urban relationships. Both of them are present as a real fact in some way. At the end, this doubled presence is shown with a synthetic and enlightening aim.
\end{abstract}

Dpto. Sociología I UNED. jadelpino@poli.uned.es

Una primera versión de este trabajo se presentó en el Grupo de Trabajo de Sociología Urbana del VII Congreso Español de Sociología celebrado en Salamanca en septiembre de 2001 bajo el título: "Globalización, redes urbanas y flujos". 


\section{PALABRAS CLAVE: KEY WORDS: \\ Sistemas Urbanos, Redes Urbanas, Urban Systems, Urban Networks, \\ Globalización. Globalisation.}

\section{LOS SISTEMAS DE CIUDADES}

Hace ya décadas que los estudiosos de la ciudad percibieron la importancia de las relaciones entre ciudades para la evolución, no sólo de las diversas urbes, sino de conjuntos socioespaciales más amplios como las regiones o los estados. Las ciudades no pueden estudiarse aisladamente en la medida en que son elementos espaciales en la organización regional o nacional de la economía, la sociedad y la política. La sociología urbana norteamericana prestó especial atención a los fenómenos de interdependencia urbana a partir de la percepción desde los años cuarenta del pasado siglo del balanceo económico que llevó al enriquecimiento de las ciudades del sur (sunbelt) en detrimento de las tradicionalmente ricas ciudades del norte (snowbelt). Se planteó entonces la posibilidad de estudiar el conjunto nacional de ciudades como un sistema, de acuerdo con la apuesta metateórica tan en boga en aquellos años de analizar cualquier fenómeno bajo el prisma de los sistemas. Desde este punto de vista, se trataba del estudio de unos elementos (las ciudades), sus atributos (la población, el empleo, la producción, etc.), y las relaciones entre ambos, y entre los elementos entre sí (Hall y Fagen, 1956, en Racionero, 1981: 16). La más conocida relación sistémica es la misteriosa ley rango-tamaño, que relaciona el número de habitantes con el rango de la ciudad en el sistema.

Desde esta perspectiva, metateóricamente sistémica pero sustancialmente enclavada dentro de los planteamientos ecológico- humanos, los dos conceptos que articulan los sistemas de ciudades son el de especialización funcional y el de jerarquía urbana ${ }^{2}$. El concepto de especialización funcional es la cara urbana del concepto de división del trabajo, que tanto peso ha tenido en el desarrollo económico moderno. Partiendo de que las actividades económicas influyen notoriamente en la distribución espacial de las poblaciones y en el mismo crecimiento de la ciudades (a menudo

No quiere decirse que las relaciones demográficas del sistema, como la ley rango-tamaño, no tengan ninguna importancia, pero calculamos que, desde el punto de vista teórico, las relaciones funcionales son mucho más importantes. Las relaciones demográficas suelen ser generalizaciones de sucesos que ocurren en la realidad, pero parece arriesgado utilizarlas como variables independientes. Por ejemplo, es dificil argumentar que el aumento de la población traerá más desarrollo a una región, mientras que es un presupuesto de la planificación, plantear localización de actividades para incrementar el desarrollo urbano. 
solapando crecimiento poblacional y económico), se entiende que las ciudades funcionan en el orden sistémico como productoras de bienes y servicios para sí mismas (sector no básico) y para el exterior (sector básico). Desde este punto de vista, en el que prima el desarrollo productivo industrial, los procesos de crecimiento urbano dentro de un sistema se producen por la lucha entre ciudades por dominar las diferentes parcelas del sector básico (ser los mayores exportadores de coches, o de aceite envasado, o de pastillas de jabón, etc.). Para Pred, comentando el caso norteamericano, las ciudades con ventajas iniciales (semilleros de innovación, estructuras institucionales implicadas) en algún sector tienden a consolidar su posición y a incrementar su umbral de crecimiento al atraer al máximo número de agentes económicos implicados en el sector. De esta manera, el desarrollo urbano se convierte en un proceso acumulativo, en el que riqueza llama a riqueza a partir de una ventaja inicial. Desde luego, diversos factores ayudan a incrementar la base económica de la ciudad: la localización respecto a los recursos necesarios para el desarrollo de su sector básico y respecto a las ciudades que constituyen su mercado; la tecnología de explotación de los recursos y los medios de transporte; el desarrollo de la economía del resto del sistema; el tamaño y composición demográfica del lugar. Por otra parte, no hay que olvidar que los sistemas son dinámicos y los cambios en los atributos de los lugares (población, tecnología, etc.) pueden producir importantes cambios en el sistema, como los producidos en el sistema urbano norteamericano.

La especialización funcional de las ciudades, las diferencias de tamaño y el distinto grado de integración generan una organización jerárquica del sistema de ciudades (Precedo, 1988: 138), si bien es la aplicación de criterios de dominación a la especialización funcional la que más poderosamente define la jerarquía en la red de asentamientos desde el punto de vista teórico. Así, cabe distinguir los criterios meramente poblacionales de los más distintivamente teóricos a la hora de establecer jerarquías urbanas. Algunos estudios tienden a establecer jerarquías a través del tamaño de las ciudades, cayendo en el error teórico (sin embargo, no empírico) de igualar crecimiento urbano e importancia en el sistema ${ }^{3}$. La aplicación de

3 Es el error de Racionero, quien, a pesar de señalar que lo que define la posición de una ciudad en un nivel y otro del sistema será la naturaleza de sus funciones dentro del sistema mundial (RACIONERO, 1980, 39), no deja de referirse en ningún momento a los volúmenes poblacionales de las ciudades, decrecientes según su lugar en la jerarquia: capitales mundiales, de subsistemas nacionales, regionales o comarcales. También el estudio de MORENO JIMÉnEZ, sobre concentración de la población y jerarquia de asentamientos, trata la jerarquización por tamaño, si bien también incluye las tendencias contraurbanizadoras del sistema estadounidense (MORENO JlMENEZ, 1987) 
un principio teórico de dominación, que fundamente el establecimiento de una jerarquía, la encontramos cuando se asigna a ciertas actividades urbanas un rango mayor que a otras. Es lo que intentó Díez Nicolás al elegir seis indicadores que sintetizaran lo que teóricamente se había establecido como funciones de dominación en un sistema social complejo como el sistema urbano español de los años sesenta (Díez Nicolás, 1972: 179-213).

En definitiva, el planteamiento de las relaciones interurbanas en términos sistémicos, en el contexto de la urbanización, muestra como proceso primordial la especialización funcional, y como producto, la jerarquía urbana. Como en la división del trabajo, el principio de la competencia obliga a la búsqueda de la eficiencia; en el caso del sistema urbano, la especialización funcional ( $y$ también la propia concentración urbana) se revela naturalmente como el modo más eficiente de lograr el máximo de beneficios con el menor costo. Este modo de analizar la realidad, refrendado por multitud de estudios empíricos ha llevado durante muchas décadas a verter ríos de tinta en torno a la localización de actividades económicas.

Tratar los asentamientos como sistemas, determinar su organización jerárquica y asistir a una creciente globalización de los sistemas sociales sugiere inmediatamente la idea de tratar los sistemas urbanos de manera jerárquica, no sólo en lo referente a su organización interna. De esta manera, se aboga por la existencia de un sistema mundial de ciudades, compuesto en un segundo nivel por subsistemas nacionales (o, simplemente, mega regionales); en un tercer nivel, de subsistemas regionales; y en un cuarto nivel, de subsistemas comarcales (Racionero, 1980: 37-41). Estos sistemas y subsistemas se articulan jerárquicamente a la hora del desarrollo económico, especialmente de la recepción de las innovaciones necesarias para el desarrollo, de manera que los grandes centros innovadores, en gran medida coincidentes con las grandes áreas metropolitanas globales, difunden las novedades productivas y organizativas a través de las cabezas de los sistemas nacionales, difundiéndose posteriormente a los centros regionales y, finalmente, a las cabezas del sistema urbano comarcal. Evidentemente, algunas partes del sistema, como los sistemas nacionales cuya cabeza es también líder mundial, experimentan procesos de difusión más rápidos que otras, como por ejemplo los estados cuyas capitales, en términos de jerarquía urbana, se sitúan como cabezas regionales de subsistemas mega regionales de menos recursos, como ocurre en los paises en vías de desarrollo.

En España, el estudio del sistema urbano, adscrito sobre todo al quehacer académico de los geógrafos, está muy ligado al estudio de la urbanización en sus aspectos demográficos, como son el tamaño y la concen- 
tración espacial de la población. No faltan, sin embargo, algunos estudios de especialización funcional aunque escasamente superan el interés descriptivo o simplemente apuntan a señalar la creciente diversificación funcional que conlleva el proceso de urbanización. Este es el interés de las pioneras investigaciones de Capel para datos de 1950. De mucho más calado es la tesis de Díez Nicolás comentada anteriormente, en la que, partiendo de los presupuestos teóricos de la Ecología Humana neoortodoxa, se propone el estudio de, por un lado, la especialización funcional y, por otro, la jerarquía urbana española a partir del concepto de dominación. Lo destacable de este intento es, como ya hemos apuntado, la fundamentación teórica de la jerarquía, en la medida en que se reconocen ciertas funciones como dominantes en los sistemas urbanos complejos; concretamente, las funciones de coordinación, control, distribución y reclutamiento. Tras la selección de los indicadores de estas funciones, se ofrece un indice de dominación y la clasificación de 240 asentamientos urbanos distinguiendo de forma arbitraria: dominantes, subdominantes, influyentes, subinfluyentes y no dominantes (Díez Nicolás, 1972).

Entre los trabajos que inciden en aspectos demográficos para estudiar la jerarquía de asentamientos urbanos merecen ser señalados dos de los publicados en los años ochenta. El primero, de Antonio Moreno Jiménez, estudia el sistema de asentamientos a partir de los cambios en la concentración urbana registrada en los censos españoles desde 1950. Las conclusiones más sobresalientes del estudio hablan de un proceso de concentración urbana en el nivel provincial desde 1950 hasta 1980 (todo el periodo analizado). La visión del conjunto nacional de asentamientos queda resumida de la siguiente manera: Los cambios económicos, técnicos, organizativos y sociales que han ocurrido, y que proseguirán, en nuestro país están conduciendo a una reorganización muy importante de la estructura jerárquica y espacial de los asentamientos, a través fundamentalmente de los movimientos migratorios generados (Moreno Jiménez, 1987: 102). Efectivamente, si miramos el asunto desde un punto de vista demográfico, es evidente que uno de los efectos del complejo proceso de modernización (donde actúan factores económicos, tecnológicos, organizativos y sociales) es el crecimiento urbano, y que éste llega a altos niveles, en contextos de un crecimiento natural no desorbitado, a través de la inmigración. Más adelante señalaremos algunas cuestiones relevantes sobre los cambios en los sistemas urbanos detectados a partir de los años sesenta en Estados Unidos y Europa, que, por la tardía modernización de nuestro país, sólo son apuntadas en este artículo como posibles escenarios futuros.

El segundo trabajo que viene a colación es el intento más sólido de divulgar el estudio de nuestro sistema urbano nacional. En La red urbana, 
Andrés Precedo trata de sintetizar los aspectos fundamentales del sistema, partiendo del proceso de urbanización y haciendo especial hincapié en su aspecto histórico: desde la formación del sistema de asentamientos en la era preindustrial, primero con la red romana de asentamientos, luego con el importante papel de la cultura islámica en la vida urbana, pasando por la articulación de la red urbana moderna con capital en Madrid, y llegando a la industrialización y el crecimiento urbano iniciado en el siglo XIX. A partir de la segunda mitad de este siglo, se comienza el proceso de urbanización ligado a la modernización que se culmina finalmente en los años sesenta y setenta del siglo $\mathrm{xx}$. Es en esta época cuando encontramos un sistema urbano trabado, en el que la interdependencia entre regiones se hace verdaderamente manifiesta, y en el que los movimientos migratorios señalados anteriormente juegan un importante papel. El recorrido histórico de Precedo ayuda a no perder de vista la importancia de los factores históricos concretos que actúan en el sistema urbano, de manera que el sistema se comunica no sólo con otros sistemas urbanos de manera jerárquica sino consigo mismo y con los subsistemas que lo conforman (y que, en el pasado, fueron autónomos), en el tiempo. El recorrido histórico realizado por Precedo termina con la urbanización postindustrial, asunto que discutiremos más adelante.

Además, Precedo traza un marco de análisis sencillo para estudiar el sistema de ciudades. En primer lugar, realiza un estudio morfológico de los elementos del sistema, con especial atención al grado de concentración urbana de las ciudades; de ahí surge la distinción entre aglomeraciones policéntricas, aglomeraciones monocéntricas y áreas urbanas concentradas. También incluye un apunte morfológico sobre el conjunto del sistema, de carácter semianular, compuesto por cuatro grandes subsistemas periféricos (eje septentrional, eje de conexión del valle del Ebro, eje del Mediterráneo Oriental y la red urbana andaluza), al que se superpone la estructura radiocéntrica que tiene a Madrid como centro, apoyada por la red nacional de transportes y comunicaciones. A continuación, se estudian las relaciones demográficas del sistema, bajo el prisma de la ley rango- tamaño que dan la conocida conclusión de que el sistema español de ciudades posee una estructura bipolar equilibrada, ajustada a una distribución jerarquizada del tipo rango-tamaño, es decir, que la anomalía más sobresaliente del sistema nacional respecto a la ley se produce por el tamaño muy superior al esperado de Barcelona, la segunda ciudad del país. Finalmente el estudio de las funciones y la jerarquía urbana, conduce a Precedo a considerar la clasificación funcional de las ciudades españolas, asumiendo la relación entre el grado de desarrollo (posición en la jerarquia) y el sector de actividad económica dominante, haciendo especial 
Hacia la comprensión del espacio urbano global: ¿Sistemas de ciudades...

hincapié en las funciones terciarias. Entre las conclusiones más interesantes del estudio empírico destacan las relaciones entre rango terciario y tamaño demográfico (sobre todo a partir de los 300.000 habitantes), entre rango terciario y desarrollo económico (en términos de renta per capita), y entre rango terciario y la importancia de su área de mercado (volumen de población, nivel de actividad y consumo), para datos de 1986.

El estudio, junto a su gran interés descriptivo y morfológico, recoge las principales fuerzas que han actuado en el sistema urbano español, añadiendo algunos datos empíricos, aunque no siempre queden claros los caminos metodológicos empleados. Quizás dispone de un débil marco teórico, algunas de cuyas distinciones no consideramos afortunadas ${ }^{4}$. Sin embargo, en lo referente al análisis del sistema urbano nacional, supera con creces el confuso intento de Racionero por señalar los parámetros del sistema (Racionero, 1980: 108-147).

\section{REDES URBANAS EN EL CONTEXTO DE LA GLOBALIZACIÓN}

Aunque hoy en día resulta tópico referirse a la globalización en todos los temas que atañen a las ciencias sociales, en el caso de los estudios urbanos, y muy especialmente en lo que se refiere a los sistemas urbanos, también resulta una cuestión ineludible.

Sucintamente, la palabra globalización denota la creación de redes, sobre todo financieras y monetarias, pero también culturales y políticas, que abarcan al mundo entero. Más en particular, hace referencia al creciente papel de las empresas multinacionales y transnacionales $y$, sobre todo, al aumento producido a lo largo de los noventa en los flujos financieros (Tortosa, 1998: 516). Puede entenderse bien como una fuerza autónoma producida por las relaciones entre los agentes (económicos, pero también sociales, culturales o políticos) que corre independientemente de los Estados respondiendo a una lógica propia, bien como una fase más del capitalismo, que parte de las zonas más desarrolladas del globo y cuya característica más sobresaliente es la creciente transferencia de capitales

4 Aunque Precedo titula su trabajo La Red Urbana, su concepto de red difiere bastante de lo que aquí se entiende. Para PRECEDo, la red urbana es la plasmación formal en el espacio geográfico (...) y en un momento determinado de tiempo [de la estructura formada por las ciudades, las funciones urbanas y sus interrelaciones] (PRECEdo, 1988, 86). Asi que la red constituye un concepto estático frente al de sistema, que sería dinámico. Aquí se defienden los conceptos de red y sistema como dos tipos de estructuras interurbanas diferentes. $Y$, curiosamente, la estructura reticular parece dar lugar, en principio, a procesos más dinámicos que los procesos sistémicos. 
desde los sectores productivos y comerciales hacia la intermediación financiera y la especulación.

En la investigación urbana ya a principios de los noventa había un importante conjunto de estudios dedicados a analizar diversos aspectos de la relación entre globalización y ciudades, tales como las ciudades globales, la globalización del crecimiento metropolitano, la globalización de los servicios a empresas, la globalización cultural, las raíces históricas de la globalización urbana, etc. A pesar de que la nueva sociología urbana había irrumpido a finales de los sesenta llamando a profundizar en las razones últimas que determinaban el fenómeno urbano, no es hasta los ochenta cuando este interés por la globalización se hace más patente. Para $A$. King, la razón del lapso de tiempo entre el desarrollo de la globalización y su análisis científico en el campo urbanístico está en que sólo cuando la base económica de las ciudades de las economias avanzadas fueron afectadas fuertemente [por la globalización], hubo muchos urbanistas dispuestos a mirar más allá de las fronteras nacionales hacia el más amplio sistema económico que las sustentaba (King, 1990: 7). Además, las explicaciones sobre la urbanización dadas hasta entonces precisaban contextos más amplios desde el punto de vista histórico y geográfico. Así que, aunque los movimientos económicos producidos en los setenta ya apuntaban claramente el desarrollo de la globalización, también fue necesario en la disciplina un cambio en la percepción de los problemas urbanos.

A la hora de establecer las relaciones entre sistemas de ciudades y globalización, resulta enormemente útil distinguir entre ciudades globales y redes globales de nodos urbanos. Como vamos a ver, en todo el debate sustantivo sobre los sistemas urbanos, dejando de lado las pesquisas sobre tamaños poblacionales y morfologías urbanas, los factores económicos parecen determinantes. Aunque unos y otros se retrotraen más o menos en el tiempo para explicar las causas de la globalización, lo que parece clara es la génesis económica de la misma ${ }^{5}$. Así, Castells parte de las transformaciones tecnológicas que han llevado al nacimiento de una economía informacional global, de manera que las actividades estratégicamente dominantes (actividades basadas en el tratamiento y procesamiento de información) funcionan globalmente en tiempo real. Aunque la

5 Aunque los más conocidos defensores de la globalización como factor determinante del urbanismo actual (FRIEDMAN, CASTELLS, SASSEN) suelen defender que la génesis de estos procesos nace como muy pronto tras la Segunda Guerra Mundial, explotando sobre todo tras la reestructuración productiva fruto de la crisis de los setenta, algunos autores entroncan con la época colonial para demostrar la continuidad de ciertas estructuras que parecen ahora novedosas para algunos (A. KING, 1990). 
economía global podría localizarse en cualquier lugar dadas las posibilidades tecnológicas, lo cierto es que existe en realidad una concentración de sectores dinámicos en algunas áreas metropolitanas. Castells y Borja señalan que para entrar en la economía global, se debe constituir un nodo urbano que contenga los siguientes elementos: gestión de servicios avanzados, aeropuerto internacional, telecomunicaciones por satélite, hoteles de lujo, asistencia secretarial en inglés, empresas financieras y consultoras, oficinas del gobierno regional y local para inversiones, y personal cualificado. Sin embargo, los autores reconocen que la emergencia de los nodos globales no depende tanto de planeamientos urbanísticos como de complejos factores internacionales sobre los que existe escaso control. Por otro lado, la razón de la concentración espacial de las actividades que rigen el sistema global está, siguiendo a Sassen, en que son (1) puntos direccionales de la organización de la economía global, (2) localizaciones clave para las empresas financieras y de servicios especializados, (3) lugares de producción, incluyendo sectores avanzados y (4) son un gran mercado para lo producido (Sassen, 1991: 3-4); a lo que se añaden factores como las fuertes inversiones inmobiliarias que se mantienen, las relaciones personales del personal altamente cualificado o las oportunidades de vida que estas ciudades ofrecen. Estos puntos de alta concentración económica (a menudo, también poblacional) son las llamadas ciudades globales.

Sin embargo, junto a las ciudades globales, que ocupan el máximo nivel en la jerarquia mundial de ciudades, Castells y Borja defienden la existencia de una red global de nodos urbanos de inferior rango que gestionan asuntos globales a menor escala, y que conectan a escala global a miuchas localidades y regiones. Lo más interesante del asunto es que las posiciones en la jerarquía no están aseguradas, de manera que las ciudades compiten por captar intereses dentro de los sectores innovadores más competitivos.

Aunque existen antecedentes en Geddes que hablan de la dirección de los más importantes negocios del mundo en una serie de ciudades ya en 1915, y durante los años sesenta y setenta se hacen estudios que tratan de detectar la influencia de las cada vez más importantes relaciones entre las grandes ciudades ${ }^{6}$, quizás son los trabajos de J. Friedmann (es-

6 Pueden citarse, por ejemplo, los trabajos de TORNQVIST sobre las relaciones entre miembros de empresas suecas usuarios del avión, y los de Pedersen, en torno a la difusión de innovaciones empresariales a través de los sistemas urbanos. TORNOVIST, G. (1970): "Contact Systems and regional Development", en Lund Studies in Geography, SEC. B, 35; Gould, P. y Tornovist, G. (1971): "Information, Innovation and Acceptance", en Lund Studies in Geography, Sec. B, 37; 
pecialmente The World City Hipotheses) los que introducen en el ámbito académico las primeras aportaciones teóricas sólidas para el estudio del fenómeno que, ya a mitad de los ochenta, se desarrollaba imparablemente: la concentración de las actividades económicas punteras y directrices en una serie de ciudades del mundo. Las hipótesis de Friedman pueden resumirse de la siguiente manera:

1. La integración de la ciudad en la economía mundial a través de las funciones asignadas por la nueva división del trabajo serán decisivas para cualquier cambio estructural que se produzca dentro de ella

2. Existen ciudades clave que el capital global utiliza como puntos base de la organización espacial y la articulación de la producción y los mercados. Los lazos resultantes permiten establecer una compleja jerarquía espacial de ciudades mundiales.

3. Las funciones de control global de las ciudades mundiales se reflejan en la estructura y dinámica de sus sectores productivos y de empleo.

4. Las ciudades mundiales son lugares privilegiados de acumulación de capital internacional.

5. Las ciudades mundiales son destino de inmigrantes de todo el mundo.

6. La formación de ciudades mundiales conlleva también las mayores contradicciones del mundo capitalista, con la polarización social y espacial.

7. El crecimiento de la ciudad mundial genera grandes costos sociales superiores a los que la tradicional capacidad fiscal de los estados no pueden hacer frente.

Las tesis de Friedman fueron criticadas por diferentes razones, especialmente por no formular una teoría sobre la red de nodos globales, más allá de la existencia de los grandes centros de poder económico mundial, y por no tener en cuenta los factores de crecimiento regionales y locales (crítica de Korff, expuesta en King, 1990: 31).

Después de las hipótesis de Friedman, las formulaciones que más fortuna han hecho sobre el ascenso de una nueva estructura mundial de ciudades han sido las de M. Castells y S. Sassen. Dada la complementariedad de ambas aportaciones, comenzaremos con las ideas de Sassen, más

Pedersen, P.O. (1971): “Innovation Diffusion within and between National Urban Systems", en Geographical Analysis. (Citados en RaCionero, 1980, 21n). 
Hacia la comprensión del espacio urbano global: ¿Sistemas de ciudades...

pegadas a la realidad a través del análisis empírico de los sectores económicos globalizados y las estructuras urbanas globales resultantes, y dejaremos para luego las sugerentes lucubraciones de Castells. Para Sassen, desde los años sesenta se producen profundas transformaciones en el sistema económico que, en lo referente al espacio, dan lugar a una organización económica espacialmente dispersa (a través de la flexibilización, subcontratación, etc.) aunque globalmente integrada. Esta nueva organización ha otorgado un papel estratégico nuevo a las principales ciudades que concentran las actividades más importantes del sistema económico citadas más arriba. Pero las ciudades globales no sólo incluyen procesos económicos sino sociales y organizativos. Sassen señala los cuatro puntos principales de su análisis, que aquí sintetizamos, enfatizando el aspecto de las jerarquías urbanas:

1. La dispersión territorial de la actividad económica crea una necesidad para la expansión del control y la dirección centralizada. Se trata de la fuerza principal que anima los procesos de surgimiento de ciudades globales.

2. La principal implicación del modelo económico sobre el sistema de ciudades es que las ciudades globales no sólo son cabezas formales del sistema sino sus cabezas sustantivas, en la medida en que los sectores importantes de la economía han pasado de ser las manufacturas y los servicios de la economía industrial a los servicios especializados (al consumidor y a las empresas) y la industria financiera, sectores que concentran el control global de la economía y se localizan en estas ciudades.

3 . El sistema o red de ciudades resultante muestra relaciones complejas con el sistema de ciudades precedente, especialmente en lo que se refiere a las relaciones entre la ciudad global y su sistema nacional, y entre diferentes ciudades globales. Aunque también son cabezas de sus redes nacionales, Nueva York, Tokio y Londres (las ciudades estudiadas sin ánimo exclusivista por Sassen) son la cúspide de una nueva jerarquía global basada en flujos (sobre todo financieros), que coordinan, a la vez que participan como escenarios principales de los mercados internacionales de compraventa de capital y pericia. La complejidad de las relaciones entre las ciudades globales las lleva a ser competitivas en el mercado, pero también a repartir funciones (por ejemplo, durante los años 80 , Tokio exportaba el capital que procesaba Londres y acababa comprándose en Nueva York), incluso a funcionar como verdaderas aliadas ante las demandas improcedentes de los estados nacionales. Por otra parte, Sassen plantea la durabilidad de un sistema como éste, donde los beneficios se computan globalmente, sin tener en cuenta las unidades sociales de antaño, como las propias ciudades, las regiones o las sociedades nacionales. 
4. Sassen destaca uno de los que se convierte en tema estrella del debate en torno a la ciudad en la época de la globalización: la dualización o polarización social, cuyo efecto más sobresaliente es la fractura en el mercado laboral entre los que alcanzan altos puestos de gran cualificación y múltiples incentivos, y quienes se ven abocados a abandonar las industrias en declive o engrosan directamente el creciente sector de servicios poco cualificados ${ }^{7}$.

Castells, expuso en 1989 sus ideas en torno a la ciudad informacional, aspecto urbano de un marco de análisis más extenso sobre las sociedades contemporáneas, que el autor desarrolló en La sociedad red a mediados de los noventa. Castells parte del cambio tecnológico para explicar los cambios en la economía, la globalización y la consiguiente transformación de la ciudad. Ya hablamos más arriba de las líneas fundamentales de su pensamiento acerca de los procesos urbanos en la nueva sociedad, el nacimiento de una red de nodos urbanos globales. Además de la visión general de los procesos urbanos, hay que señalar la encomiable labor investigadora de Castells sobre los medios de innovación (especialmente su estudio sobre las tecnópolis como formas urbanas que albergan los medios de innovación) y su papel en la nueva economía informacional. Nos centraremos ahora en las consecuencias espaciales ${ }^{8}$ de esta nueva estructura del sistema-red de ciudades. En primer lugar, para Castells, la ciudad global no es un lugar, sino un proceso. Un proceso mediante el cual los centros de producción y consumo de servicios avanzados y sus sociedades locales auxiliares se conectan en una red global en virtud de los flujos de información, mientras que a la vez restan importancia a las

\footnotetext{
El debate en torno a la polarización social producida por la globalización es estudiado tanto por CASTELLS como por SASSEN. Mucho de éste debate se circunscribe a la conformación interna de las ciudades, al advenimiento de nuevos procesos de segregación espacial alimentados por la nueva economia. En 2000, apareció un interesante trabajo monográfico sobre los efectos de la globalización en el espacio interno de las ciudades que merece ser leido por la riqueza de contextos analizados y por las conclusiones obtenidas por los editores, quienes, a pesar de partir de la hipótesis de que la globalización apoyaba la dualización espacial, terminan concluyendo que la va* riedad de sifuaciones impide ver una clara relación entre globalización y dualización, aunque sí parece existir un incremento de desigualdades que, en ningún caso, parece irreversible si se pone voluntad en ello. MARCUSE, P. y VAN KEMPEN (eds.) (2000): Globalizing cities. A new spatial order? Blackwell.

8 Nos referimos a las consecuencias espaciales en los términos en que entiende CASTELLS el espacio, un espacio material socialmente definido, cuya definición propuesta en la La Question Urbaine (la crítica de la sociología urbana de la Escuela de Chicago que contribuyó decisivamente a la creación de una sociología crítica de la ciudad) sigue recogiendo el autor en La era de la información: El espacio es un producto material en relación con otros productos materiales -incluida la gente- que participan en relaciones sociales determinadas [históricamente] y que asignan al espacio una forma, una función y un significado social (CASTELLS, 1997, 444).
} 
Hacia la comprensión del espacio urbano global: ¿Sistemas de ciudades...

conexiones con sus entornos territoriales (Castells, 1997: 419). Las posibilidades del hogar electrónico (el teletrabajo, el telecomercio, la telesanidad, la educación a distancia, etc.) y la cada vez mayor importancia de la conexión telemática superando la continuidad territorial que definía la ciudad, son consecuencias de este proceso. Más allá incluso, es consecuencia del nuevo espacio la reestructuración de los espacios urbanos, con centros de negocios homogéneos en la medida en que forman parte de la red intermetropolitana, los espacios de segregación estructurados bajo las mismas pautas en todos los lugares con más o menos intensidad según sea el grado de integración en la red global, etc.

La tesis fuerte de Castells es que existe una nueva forma espacial característica de la sociedad red, el espacio de los flujos (en contraposición del espacio de los lugares), que es definido como la organización material de las prácticas sociales en tiempo compartido que funcionan a través de los flujos (Castells, 1997: 445), en el entendido de que los flujos son las secuencias de intercambio e interacción determinadas, repetitivas y programables entre las posiciones físicamente inconexas que mantienen los actores sociales en las estructuras económicas, políticas y simbólicas de la sociedad (Castells, 1997: 445). Aunque el antiguo espacio, el de los lugares, sigue existiendo y es portador de importantes cualidades físicas y simbólicas que ayudan a conformar identidades, el espacio de los flujos es dominante, en la medida en que articula a las elites (cosmopolitas, globales) y desarticula a las masas (locales). Dos de los mecanismos que producen este distanciamiento, para la seguridad de las elites, es el aislamiento simbólico y físico de estos sectores sociales y la creación de estilos de vida globales, uno de los aspectos más sobresalientes de la globalizaciún por su popularidad: la homogeneización de aeropuertos, hoteles, de procesos organizativos, etc.

\section{3. ¿SISTEMAS DE CIUDADES O REDES?}

A pesar de que los autores citados en el anterior apartado son generosos a la hora de apoyar sus ideas en estudios empíricos, es difícil encontrar datos que avalen sin paliativos la existencia de una red mundial de ciudades. Desde luego, existen indicadores que prueban que muchas de las tendencias apuntadas son reales, como el predominio de los sectores avanzados en las ciudades económicamente más dinámicas o los niveles más altos de relación entre las ciudades más importantes del globo. Lo que sí parece afianzarse definitivamente en los estudios urbanos es la idea de que las ciudades no pueden estudiarse aisladamente, sino en relación al resto de ciudades próximas, funcional y/ o espacialmente. En re- 
ferencia al espacio geográfico, el estudio de las ciudades tiende a la regionalización, en dos sentidos. Regionalización de las unidades de análisis, elementos del sistema o nodos de la red, de las ciudades en definitiva. Por lo que atañe a nuestro país, la metropolización es un hecho insoslayable a la hora de interpretar los procesos inter e intra urbanos, lo que exige como mínimo superar unidades administrativas como los municipios. Por otro lado, la regionalización de los estudios urbanos también se refiere a la necesidad de estudiar las relaciones interurbanas teniendo en cuenta la apertura de posibilidades para cada nodo de la red, cuyo tamaño poblacional comienza a no ser sinónimo de posición de más o menos altura en la jerarquía. El término región comprende un vastísimo rango de niveles de análisis, desde la comunidad autónoma hasta el continente, pero deben ser el objeto y los objetivos de la investigación los que determinen a qué nivel debemos referirnos.

Para terminar estas reflexiones en torno a las relaciones interurbanas, parece obligada la presentación de una síntesis como modesta aportación a la comprensión de los fenómenos estudiados. Conclusión de este trabajo que, debe recalcarse, es provisional, pues quien suscribe lo entiende como una parada en el camino y no como llegada a puerto. Siguiendo el modelo de racionalización comprensiva weberiana, presentamos un par de tipos ideales de estructuras interurbanas, basadas en la realidad ${ }^{9}$ pero nunca iguales a ella, tipos puros a los que la realidad tiende sin confundirse, presentando, más o menos, los rasgos que se proponen (Tabla 1) ${ }^{10}$.

El primer tipo propuesto es el sistema, primera forma bajo la que se teorizó sobre las relaciones interurbanas y sus efectos territoriales, como vimos en el primer apartado. Los sistemas se caracterizan por el protagonismo de los lugares, las ciudades, y por atender a un principio de jerarquía. El modelo christalleriano de lugares centrales es un acabado modelo de este tipo de estructura interurbana. Además, el modelo sistémico ha sido el predominante. Por su parte, el modelo reticular surge con la reestructuración económica mundial producida desde la década de los años setenta. En el nuevo modelo, el protagonismo se cede a los flujos, más

9 Los tipos presentados están basados en las ideas de los autores expuestos y en las propias del autor, no en trabajos empíricos, propios o ajenos. Sólo en el supuesto de que estas ideas representen la realidad como es, estaremos haciendo tipología basada en el fenómeno que quere. mos analizar. Si estas teorias no responden a la realidad, simplemente estaremos mostrando dos tipos de teorización sobre los sistemas de ciudades, lo cual no deja de ser interesante, aunque, honradamente, no es el objetivo de este trabajo.

10 No se pretende ser exhaustivo, pero, a nuestro parecer, se mencionan los rasgos definitorios de cada tipo 
que a los lugares, y el principio que comienza a regir puede llegar a ser la horizontalidad. Los flujos determinan más las relaciones interurbanas que los propios atributos de las ciudades. El espacio vivido deja de coincidir exactamente con el espacio geográfico; el ejemplo más claro lo encontramos en las redes telemáticas, donde el espacio vivido ya no es el lugar sino la red de flujos. En cuanto a la horizontalidad, ésta suele plantearse teóricamente, porque es una de las propiedades más interesantes de algunas redes (como Internet), pero quizás sea difícil encontrar apoyo empírico que la sustente como principio de la estructura interurbana. Brunet señala que puede detectarse este principio de horizontalidad (o de desvanecimiento del jerárquico) en el nacimiento de redes de connivencia (ciudades, sin importar el tamaño, que cooperan en un proyecto común para fomentar un grupo de presión en torno a un tema concreto o una línea de investigación) (Brunet, 2000).

Morfológicamente, la estructura sistémica produce la concentración de la población en lugares centrales. La metropolización (forma sobresaliente del crecimiento urbano) y su relación con el desarrollo ha sido una de las grandes discusiones de los estudiosos de los sistemas urbanos. Aunque la metropolización y la modernización de las sociedades en su conjunto parecen ir de la mano, también es claro que ha producido importantes desequilibrios territoriales. Sin embargo, los procesos de crecimiento urbano estadounidenses en la segunda mitad del siglo xx llevaron a algunos sociólogos a hablar de procesos de contraurbanización. Así, Hall y Hay trataron de probar a principios de los ochenta si estas tendencias se daban también en Europa, testando las siguientes hipótesis: que el crecimiento urbano se dirigía desde las áreas metropolitanas (1) hacia los lugares de inenor jerarquía del sistema; (2) dentro de las áreas metropolitanas, hacia los cinturones; (3) hacia zonas rurales; (4) hacia las ciudades de reciente industrialización y urbanización, centradas en los servicios (Hall y Hay, 1980: 27). Estas nuevas formas de urbanización, que, por cierto, no fueron encontradas con precisión para Europa en aquel estudio, se parecen más a la morfología producida por la estructura reticular. De hecho, en relación al sistema de ciudades, podía interpretarse que la contraurbanización era producto de la generación de deseconomías externas y el deterioro de la calidad de vida en las áreas metropolitanas, y se apoyaba en las cada vez más potentes redes de comunicaciones, siguiendo los modelos organizativos empresariales (Moreno Jiménez, 1987: 103-104). La ciudad postindustrial aparece entonces, desde el prisma norteamericano, como un continuo urbano sin centro, imagen muy usada por la literatura post- moderna, el decadente, desintegrado y caótico Los Angeles 2019 de Blade Runner (Lyon, 1994: 11-15). Sin ir tan lejos, proponemos, para la estruc- 
tura en red, la morfología urbana del continuo, pero somos conscientes de que los procesos europeos no demuestran con claridad esta tendencia (Wegener y Kunzman, 1996; Demateis, 1996; Hall, 2000). Ni tampoco la belleza inimitable de nuestras ciudades.

En cuanto a la estructura intraurbana, sólo señalaremos que la estructura sistémica ha producido enormes desequilibrios dentro de las ciudades, además de entre las ciudades. Sin embargo, la novedad de las redes es que aparecen fuerzas globales en la estructuración interna de la ciudad. Siguiendo a Castells, las fuerzas globales que actúan sobre el espacio metropolitano en la era informacional producen también estructuras urbanas segregadas ${ }^{11}$. Así encontramos en las ciudades europeas un centro de negocios, núcleo de la actividad global, auténtico nodo físico de la red urbana; barrios burgueses representativos que acogen residencialmente a la elite gestora, tecnócrata y política; suburbios tradicionales, lugares ligados a su historia, actuando como espacios defensivos frente al imparable predominio de los flujos; urbanizaciones para la clase media profesional, debatida entre la suburbanización y los problemáticos centros urbanos; ghettos de inmigrantes en diferentes lugares urbanos (centros, lugares de intercambio de transportes) (Castells, 1997: 434-436). Se trata de las consecuencias locales, en el espacio geográfico, de la nueva estructura. Aunque los lugares ven reducida su importancia en la estructura reticular de ciudades, es evidente que siguen y seguirán ocupando gran parte del espacio vivido por las personas; lo que importa a nuestro análisis es que la conformación de los lugares está estrechamente relacionada con la red de flujos de ascendencia global.

Finalmente, dos apuntes sobre política, en dos sentidos bien diferentes (policy y Politics). En cuanto a la planificación urbana, encontramos en los sistemas de ciudades a la producción como objetivo central para incrementar la base económica urbana, reforzando la posición de la ciudad e incrementando el nivel competitivo del sistema, su desarrollo. La política en la estructura reticular de ciudades parece ir orientada, sin embargo, hacia el servir. No se trata tanto del ascenso de la economía de servicios, como del giro político que las ciudades están realizando, poniendo como objetivo atajar flujos. Las políticas de marketing urbano son el elemento más destacado de esta forma de entender el desarrollo. Uno de los elementos que une a las estructuras interurbanas aquí expuestas es la competitividad como animador al desarrollo tanto del sistema de ciudades

11 Véase nota 7 de este trabajo. 
como de la red, lo que, de lejos, llama a considerar las reflexiones ecológicas sobre las relaciones entre el hombre y el medio.

Estas estructuras interurbanas tienen consecuencias políticas importantes, tanto en lo referente al poder (quién toma las decisiones) como a la legitimación (cómo se acatan las decisiones). Para la estructura sistémica, los actores locales tienen fuerza para activar en gran medida las capacidades de la urbe; pueden presionar sobre las autoridades regionales y nacionales, y éstas a su vez se comportan como entidades locales, identificadas con un lugar, sea éste más o menos grande. En estos actores recae la mayor parte de la responsabilidad; $y$, aunque exista insuficiencia democrática (pues muchos ciudadanos ni deciden ni tan siquiera tienen acceso a los lugares de decisión), se puede localizar la responsabilidad, aunque sólo sea para culpabilizar a los políticos. En la red, las actuaciones no son fácilmente imputables a ninguna autoridad. Fuerzas transnacionales, que el ciudadano no localiza, parecen determinar el destino de los lugares, porque es el espacio de los flujos el que predomina. La red, sobre todo si se afianza el principio no jerárquico, permite a cualquiera llegar hasta todos los nodos (de la red urbana, social, política, cultural...), pero la responsabilidad está diluida y no se conoce a quién dirigirse o imputársela. La localización fuera de nuestro radio de acción de las fuerzas que determinan los procesos urbanos es, probablemente, el mayor problema al que se enfrenta la emergente estructura urbana. No renunciar al sentido político del hombre, a la ciudadanía, es sin duda el mejor camino de solución.

Tabla 1. Tipos de estructuras interurbanas

\begin{tabular}{lll}
\hline Estructura interurbana & \multicolumn{1}{c}{ SISTEMA } & \multicolumn{1}{c}{ RED } \\
\hline Elemento principal & Lugar & Flujo \\
Principio & Jerarquía & Horizontalidad \\
Proceso morfológico & Metropolización & Continuo urbano \\
Estructura intraurbana & Segregación local & Segregación global \\
Politica: planeamiento & Producir base económica & Atajar flujos (servir) \\
Politica: legitimación & Actores locales & Fuerzas transnacionales \\
& (Local) & (Global (lo local en función \\
& & de lo global)) \\
\hline
\end{tabular}

\section{BIBLIOGRAFIA}

Bielza de Ory, V. y de Miguel González, R. (2000): “Reestructuración económico- territorial de las grandes ciudades y nuevos enfoques para su estudio", en AA.VV. Lecturas geográficas. Homenaje a José Estébanez Álvarez, Tomo II, Madrid, Editorial Complutense, págs. 9831003. 
BONAVERO, P. y CONTI, S. (1996): “New technological paradigm, urban identity and metropolitan networks in Europe", en PUMAIN, D. y SAINT-JULIEN, T., Urban Networks in Europe, París, John Libbey e INED, págs. 47-65.

BRUnet, R. (2000): “Des villes comme Lleida. Place et perspectives des villes moyennes en Europe", en BELLET, C. y LLOP, J. M. (eds.), Ciudades intermedias. Urbanización y sostenibilidad, Lérida, Ed. Milenio, págs. 109-124.

CASTELLS, M. (1995): La ciudad informacional. Tecnologías de la información, reestructuración económica y el proceso urbano regional, Madrid, Alianza.

- (1997): La era de la información. Economía, Sociedad y Cultura. Vol. I, La sociedad red, Madrid, Alianza.

- y BORJA, J. (1997): Local y global, Taurus, Madrid.

DEMATEIS, G. (1996): "Towards a unified metropolitan urban system in Europe: core centrality versus network distributed centrality", en PUMAIN, D. y SAINT- JULIEN, T., Urban Networks in Europe, París, John Libbey e INED, págs. 19-28

Díez Nicolás, J. (1972): Especialización funcional y dominación urbana en la España urbana, Madrid, Guadarrama.

Friedman, J. (1986): "The world city hypothesis", en Development and Change, 17 (1), $69-83$.

HaLL, P. (2000): "Redefining Europe's cities", en BELLET, C. y LLOP, J. M. (eds.), Ciudades intermedias. Urbanización y sostenibilidad, Lérida, Ed. Milenio, págs. 93-108.

- y HAY, D. (1980): Growth Centres in the European Urban System, Heynemann Educational Books.

KING, A. D. (1990): Global cities. Post-Imperialism and the Internationalization of London, Routledge.

LyON, D. (1994): Postmodernidad, Madrid, Alianza.

MARCuSE, P. y VAN KeMPEN, R. (eds.) (2000): Globalizing cities. A new spatial order?, Blackwell.

MORENO JIMÉNEZ, A. (1987): “Concentración de la población y jerarquía de asentamientos en España. Evolución y perspectivas", en Estudios Territoriales, 24, págs. 77-108.

Precedo Ledo, A. (1988): La red urbana, Madrid, síntesis.

RACIONERO, L. (1980): Sistemas de ciudades y ordenación del territorio, Madrid, Alianza.

SASSEN, S. (1991): The global city. New York, London, Tokyo Princeton, Princeton University Press.

SCHWAB, W. A. (1992): "The system of cities", en Sociology of cities, Prentice Hall.

Tortosa, J. M. (1998): “Mundialización», en Giner, S.; LAMO de Espinosa, E.; TorRes, C. (eds.) Diccionario de Sociologia Madrid, Alianza, págs. 515-516

WEGENER, M. y KUNZMAN, K. (1996): "New spatial patterns of European metropolization" en Pumain, D. y SAINT-JULIEN, T., Urban Networks in Europe, París, John Libbey e INED, págs. 7-17. 